\title{
ON ASYMPTOTICALLY GENERALIZED STATISTICAL EQUIVALENT SEQUENCES VIA IDEALS
}

\author{
VIJAY KUMAR AND ARCHANA SHARMA
}

\begin{abstract}
For an admissible ideal $\mathscr{I} \subseteq \mathscr{P}(\mathbb{N})$ and a non-decreasing real sequence $\lambda=\left(\lambda_{n}\right)$ tending to $\infty$ with $\lambda_{n+1} \leq \lambda_{n}+1, \lambda_{1}=1$, the objective of this paper is to introduce the new notions $\mathscr{I}$-statistically equivalent, $\mathscr{I}-[V, \lambda]$-equivalent and $\mathscr{I}-\lambda$-statistically equivalent. which are natural combinations of the definitions for asymptotically equivalent, statistical limit, $\lambda$-statistical limit and $\mathscr{I}$-limit for number sequences. In addition, some relations among these new notions are also obtained.
\end{abstract}

\section{Introduction}

The notion of statistical convergence of sequences of numbers was introduced by Fast [2] and Schonberg [17] independently. Over the years and under different names statistical convergence has been discussed in the theory of Fourier analysis, Ergodic Theory and Number Theory. Later on, it was further investigated from the sequence space point of view and linked with summability theory by Connor [1], Fridy [4], Maddox [8], Šalát [15], Tripathy [18] and many others. For more recent works on statistical convergence we also refer, Rath and Tripathy [14], Tripathy and Sen ([19], [21]), Tripathy [20], Tripathy and Sarma [22], Tripathy and Baruah [23]. In last few years, many generalizations of statistical convergence have appeared and are found helpful in the study of the strong integral summability theory. Mursaleen [10] used the notion of $(V, \lambda)$-summability to generalize the concept of statistical convergence in term of $\lambda$-statistical convergence and investigated some of its properties. Kostyrko et al. [5] generalized statistical convergence with the help of an admissible ideal $\mathscr{I}$ of subsets of $\mathbb{N}$, the set of positive integers and called it $\mathscr{I}$-convergence. This new type of convergence has been further investigated from different aspect by Tripathy and Hazarika ([24], [25], [27], [28]), Tripathy and Mahanta [26], and many others. Quite recently, Savaş et al. [16] unified the notions of statistical convergence, $\mathscr{I}$-convergence and $\lambda$-statistical convergence to introduce new

Corresponding author: Vijay Kumar.

2010 Mathematics Subject Classification. 40A05; 40A35; 40G05.

Key words and phrases. Asymptotically equivalent sequences; statistical convergence; $\lambda$ - statistical convergence and ideal convergence. 
concepts of $\mathscr{I}$-statistical convergence, $\mathscr{I}-\lambda$-statistical convergence and investigated some of their consequences.

On the other side, Pobyvanets [13] introduced the concept of asymptotically regular ma$\operatorname{trix} A$, which preserve the asymptotic equivalence of two non-negative sequences, that is $x \sim y$ implies $A x \sim A y$. Subsequently, Marouf [9] and Li [7] studied the relationships between the asymptotic equivalence of two sequences in summability theory and presented some variations of asymptotic equivalence. Patterson [11] extended these concepts by presenting an asymptotically statistically equivalent analog of these definitions and natural regularity conditions for non-negative summability matrices. Later, these ideas were extended to lacunary sequences by Patterson and Savaş in [12]. In present work, we introduce some new notions $\mathscr{I}$-statistically equivalent, $\mathscr{I}-[V, \lambda]-$ equivalent, $\mathscr{I}-\lambda$ - statistically equivalent sequences for number sequences and investigated some relations among these new notions.

\section{Preliminaries}

For easy understanding of the material incorporated in this paper, we recall some basic definitions and results.

Definition $2.1([2])$. A number sequence $x=\left(x_{k}\right)$ is said to be statistically convergent to a number $L$ provided that for every $\epsilon>0$,

$$
\lim _{n \rightarrow \infty} \frac{1}{n}\left|\left\{k \leq n:\left|x_{k}-L\right| \geq \epsilon\right\}\right|=0,
$$

where vertical bars denote the cardinality of the enclosed set. In this case we write $S-\lim _{k \rightarrow \infty} x_{k}=$ $L$ or $x_{k} \rightarrow L(S)$.

Further, for any non-decreasing sequence $\lambda=\left(\lambda_{n}\right)$ of real numbers tending to $\infty$ with $\lambda_{n+1} \leq \lambda_{n+1}, \lambda_{1}=1$, the generalized de la Valée-Pousin mean is defined by

$$
t_{n}(x)=\frac{1}{\lambda_{n}} \sum_{k \in I_{n}} x_{k}
$$

where $I_{n}=\left[n-\lambda_{n}+1, n\right]$.

A sequence $x=\left(x_{k}\right)$ is said to be $(V, \lambda)$-summable to a number $L$ (see [6]) if $t_{n}(x) \rightarrow L$ as $n \rightarrow \infty$. If we take $\lambda_{n}=n$, then $(V, \lambda)$-summability reduces to $(C, 1)$-summability. Let,

and

$$
[C, 1]=\left\{x=\left(x_{n}\right): \exists L \in \mathbb{R}, \quad \lim _{n \rightarrow \infty}\left(\frac{1}{n} \sum_{k=1}^{n}\left|x_{k}-L\right|\right)=0\right\}
$$

$$
[V, \lambda]=\left\{x=\left(x_{n}\right): \exists L \in \mathbb{R}, \lim _{n \rightarrow \infty}\left(\frac{1}{\lambda_{n}} \sum_{k \in I_{n}}\left|x_{k}-L\right|\right)=0\right\} .
$$


Definition 2.2 ([10]). Let $\lambda=\left(\lambda_{n}\right)$ be a non-decreasing sequence of reals tending to $\infty$ with $\lambda_{n+1} \leq \lambda_{n}+1, \lambda_{1}=1$. A sequence $x=\left(x_{k}\right)$ of numbers is said to be $\lambda$-statistically convergent to $L$ provided that for every $\varepsilon>0$,

$$
\lim _{n \rightarrow \infty} \frac{1}{\lambda_{n}}\left|\left\{k \in I_{n}:\left|x_{k}-L\right| \geq \varepsilon\right\}\right|=0 .
$$

In this case we write $S_{\lambda}-\lim _{k \rightarrow \infty} x_{k}=L$ or $x_{k} \rightarrow L\left(S_{\lambda}\right)$.

We next recall the terminology of $\mathscr{I}$-convergence which is one of the main notions that we need in the sequel.

For any non-empty set $X$, let $\mathscr{P}(X)$ denotes the power set. A family $\mathscr{I} \subseteq \mathscr{P}(X)$ is said to be an ideal in $X$ if (i) $\varnothing \in \mathscr{I}$; (ii) $A, B \in \mathscr{I}$ imply $A \cup B \in \mathscr{I}$; (iii) $A \in \mathscr{I}, B \subset A$ imply $B \in \mathscr{I}$.

A non-empty family $\mathscr{F} \subseteq \mathscr{P}(X)$ is said to be a filter in $X$ if

(i) $\varnothing \notin \mathscr{F}$;

(ii) $A, B \in \mathscr{F}$ imply $A \cap B \in \mathscr{F}$;

(iii) $A \in \mathscr{F}, B \supset A$ imply $B \in \mathscr{F}$.

An ideal $\mathscr{I}$ is said to be non-trivial if $\mathscr{I} \neq\{\varnothing\}$ and $X \notin \mathscr{I}$. A non-trivial ideal $\mathscr{I}$ is called admissible if it contains all the singleton sets. If $\mathscr{I}$ is a non-trivial ideal on $X$, then $\mathscr{F}=$ $\mathscr{F}(\mathscr{I})=\{X-A: A \in \mathscr{I}\}$ is a filter on $X$ and conversely. The filter $\mathscr{F}(\mathscr{I})$ is called the filter associated with the ideal $\mathscr{I}$.

For further study, we take $X=\mathbb{N}$ and for any set $A, A^{C}$ denotes the complement of $A$.

Definition 2.3 ([5]). Let $\mathscr{I} \subseteq \mathscr{P}(\mathbb{N})$ be a non-trivial ideal. A number sequence $x=\left(x_{k}\right)$ is said to be $\mathscr{I}$-convergent to a number $L$ provided that for each $\epsilon>0$,

$$
A(\epsilon)=\left\{k \in \mathbb{N}:\left|x_{k}-L\right| \geq \epsilon\right\} \in \mathscr{I} .
$$

In this case we write $\mathscr{I}-\lim _{k \rightarrow \infty} x_{k}=L$.

Definition 2.4 ([16]). Let $\mathscr{I} \subseteq \mathscr{P}(\mathbb{N})$ be a non-trivial ideal. A sequences $x=\left(x_{k}\right)$ is said to be $\mathscr{I}$ - statistically convergent to $L$ provided that for each $\epsilon>0$ and every $\delta>0$,

$$
\left\{n \in \mathbb{N}: \frac{1}{n}\left|\left\{k \leq n:\left|x_{k}-L\right| \geq \epsilon\right\}\right| \geq \delta\right\} \in \mathscr{I} .
$$

In this case we write $\mathscr{I}-S-\lim _{k \rightarrow \infty} x_{k}=L$ or $x_{k} \rightarrow L(\mathscr{I}-S)$.

Definition 2.5 ([16]). Let $\mathscr{I} \subseteq \mathscr{P}(\mathbb{N})$ be a non-trivial ideal. A sequences $x=\left(x_{k}\right)$ is said to be $\mathscr{I}-\lambda$ - statistically convergent to $L$ provided that for each $\epsilon>0$ and every $\delta>0$,

$$
\left\{n \in \mathbb{N}: \frac{1}{\lambda_{n}}\left|\left\{k \in I_{n}:\left|x_{k}-L\right| \geq \epsilon\right\}\right| \geq \delta\right\} \in \mathscr{I} .
$$

In this case we write $\mathscr{I}-S_{\lambda}-\lim _{k \rightarrow \infty} x_{k}=L$ or $x_{k} \rightarrow L\left(\mathscr{I}-S_{\lambda}\right)$. 
Definition 2.6 ([9]). Two non-negative sequences $x=\left(x_{k}\right)$ and $y=\left(y_{k}\right)$ are said to be asymptotically equivalent of multiple $L$ provided that

$$
\lim _{k \rightarrow \infty} \frac{x_{k}}{y_{k}}=L
$$

(denoted by $x \sim y$ ) and simply asymptotically equivalent if $L=1$.

Patterson [11] presented a natural combination of Definitions 2.1 and 2.6 to introduce the concept of asymptotically statistically equivalence as follows.

Definition 2.7. The two non-negative sequences $x=\left(x_{k}\right)$ and $y=\left(y_{k}\right)$ are said to be asymptotically statistically equivalent of multiple $L$ provided that for every $\epsilon>0$, we have

$$
\lim _{n \rightarrow \infty} \frac{1}{n}\left|\left\{k \leq n:\left|\left(\frac{x_{k}}{y_{k}}\right)-L\right| \geq \epsilon\right\}\right|=0,
$$

(denoted by $x \sim^{S_{L}} y$ ) and simply asymptotically statistical equivalent if $L=1$. Let $S_{L}$ denotes the set of all sequences $x=\left(x_{k}\right)$ and $y=\left(y_{k}\right)$ such that $x \sim^{S_{L}} y$.

Now we give some new definitions which are natural combinations of Definitions 2.4, 2.5 and 2.6.

\section{Main results}

Definition 3.1. Let $\mathscr{I} \subseteq \mathscr{P}(\mathbb{N})$ be a non-trivial ideal. The two non-negative sequences $x=\left(x_{k}\right)$ and $y=\left(y_{k}\right)$ are said to be asymptotically $\mathscr{I}$-statistically equivalent of multiple $L$ provided that for each $\epsilon>0$ and every $\delta>0$,

$$
\left\{n \in \mathbb{N}: \frac{1}{n}\left|\left\{k \leq n:\left|\left(\frac{x_{k}}{y_{k}}\right)-L\right| \geq \epsilon\right\}\right| \geq \delta\right\} \in \mathscr{I} .
$$

(denoted by $x \sim^{S_{(\mathscr{I})}} y$ ) and simply asymptotically $\mathscr{I}$-statistically equivalent if $L=1$.

Let $S^{L}(\mathscr{I})$ denotes the set of all sequences $x=\left(x_{k}\right)$ and $y=\left(y_{k}\right)$ such that $x \sim^{S^{L}(\mathscr{I})} y$.

Definition 3.2. Let $\mathscr{I} \subseteq \mathscr{P}(\mathbb{N})$ be a non-trivial ideal. The two non-negative sequences $x=\left(x_{k}\right)$ and $y=\left(y_{k}\right)$ are said to be asymptotically $\mathscr{I}-[V, \lambda]$-equivalent of multiple $L$ provided that for every $\delta>0$,

$$
\left\{n \in \mathbb{N}: \frac{1}{\lambda_{n}} \sum_{k \in I_{n}}\left|\left(\frac{x_{k}}{y_{k}}\right)-L\right| \geq \delta\right\} \in \mathscr{I},
$$

(denoted by $x \sim^{[V, \lambda]^{L(\mathscr{A})}} y$ ) and simply asymptotically $\mathscr{I}-[V, \lambda]$-equivalent if $L=1$.

Let $[V, \lambda]^{L}(\mathscr{I})$ denotes the set of all sequences $x=\left(x_{k}\right)$ and $y=\left(y_{k}\right)$ such that $x \sim^{[V, \lambda]^{L(\mathscr{I})}} y$. 
Definition 3.3. Let $\mathscr{I} \subseteq \mathscr{P}(\mathbb{N})$ be a non-trivial ideal. The two non-negative sequences $x=\left(x_{k}\right)$ and $y=\left(y_{k}\right)$ are said to be asymptotically $\mathscr{I}-[C, 1]$-equivalent of multiple $L$ provided that for every $\delta>0$,

$$
\left\{n \in \mathbb{N}: \frac{1}{n} \sum_{k=1}^{n}\left|\left(\frac{x_{k}}{y_{k}}\right)-L\right| \geq \delta\right\} \in \mathscr{I},
$$

(denoted by $x \sim^{[C, 1]^{L(\mathscr{I})}} y$ ) and simply asymptotically $\mathscr{I}-[C, 1]$-equivalent if $L=1$.

Let $[C, 1]^{L}(\mathscr{I})$ denotes the set of all sequences $x=\left(x_{k}\right)$ and $y=\left(y_{k}\right)$ such that $x \sim^{[C, 1]^{L(\mathscr{A})}} y$.

Definition 3.4. Let $\mathscr{I} \subseteq \mathscr{P}(\mathbb{N})$ be a non-trivial ideal. The two non-negative sequences $x=\left(x_{k}\right)$ and $y=\left(y_{k}\right)$ are said to be asymptotically $\mathscr{I}-\lambda$ statistically equivalent of multiple $L$ provided that for each $\epsilon>0$ and every $\delta>0$,

$$
\left\{n \in \mathbb{N}: \frac{1}{\lambda_{n}}\left|\left\{k \in I_{n}:\left|\left(\frac{x_{k}}{y_{k}}\right)-L\right| \geq \epsilon\right\}\right| \geq \delta\right\} \in \mathscr{I},
$$

(denoted by $x \sim_{\lambda}^{L}(\mathscr{I})$ y) and simply asymptotically $\mathscr{I}-\lambda$ statistically equivalent if $L=1$.

Let $S_{\lambda}^{L}(\mathscr{I})$ denotes the set of all sequences $x=\left(x_{k}\right)$ and $y=\left(y_{k}\right)$ such that $x \sim_{\lambda}^{L}(\mathscr{I}) y$.

Remark 3.1. (i) If we take $\mathscr{I}=\mathscr{I}_{f}=\{A \subseteq \mathbb{N}$ : A is finite set $\}$, then the asymptotically $\mathscr{I}$-statistically equivalence, $\mathscr{I}-[V, \lambda]$-equivalent and $\mathscr{I}-\lambda$ statistically equivalence of sequences respectively coincides with their statistically equivalence, $[V, \lambda]$-equivalence and $\lambda$ - statistically equivalence.

(ii) For the choice $\lambda_{n}=n$, Definition 3.1 coincides with Definition 3.4 whereas Definition 3.2 coincides with Definition 3.3.

Theorem 3.1. Let $\mathscr{I} \subseteq \mathscr{P}(\mathbb{N})$ be an admissible ideal and $\lambda=\left(\lambda_{n}\right)$ be any non-decreasing real sequence tending to $\infty$ with $\lambda_{n+1} \leq \lambda_{n}+1, \lambda_{1}=1$, then we have the following:

(i) $x \sim^{[V, \lambda]^{L(\mathscr{I})}} y$ implies $x \sim_{\lambda}^{L}(\mathscr{I})$ y and the inclusion $[V, \lambda]^{L}(\mathscr{I})$ and $S_{\lambda}^{L}(\mathscr{I})$ is proper.

(ii) If $x=\left(x_{k}\right), y=\left(y_{k}\right) \in \ell_{\infty}$ such that $x \sim_{\lambda}^{S_{\lambda}(\mathscr{I})} y$, then $x \sim^{[V, \lambda]^{L_{(\mathscr{I})}}} y$ and hence $x \sim^{[C, 1]^{L_{(\mathscr{I})}}} y$ provided that $\left(\frac{x_{k}}{y_{k}}\right)$ is not eventually constant.

(iii) $S_{\lambda}^{L}(\mathscr{I}) \cap \ell_{\infty}=[V, \lambda]^{L}(\mathscr{I}) \cap \ell_{\infty}$. Here $\ell_{\infty}$ denotes the class of bounded sequences.

Proof. (i) Suppose $x=\left(x_{k}\right)$ and $y=\left(y_{k}\right)$ be two sequences such that $x \sim^{[V, \lambda]^{L(\mathscr{C})}} y$. We show that $x \sim_{\lambda}^{L}(\mathscr{I}) y$. Let $\epsilon>0$. Since

$$
\sum_{k \in I_{n}}\left|\left(\frac{x_{k}}{y_{k}}\right)-L\right| \geq \sum_{k \in I_{n} \text { and }\left|\left(\frac{x_{k}}{y_{k}}\right)-L\right| \geq \epsilon}\left|\left(\frac{x_{k}}{y_{k}}\right)-L\right| \geq \epsilon .\left|\left\{k \in I_{n}:\left|\left(\frac{x_{k}}{y_{k}}\right)-L\right| \geq \epsilon\right\}\right|,
$$

so for a given $\delta>0$,

$$
\frac{1}{\lambda_{n}}\left|\left\{k \in I_{n}:\left|\left(\frac{x_{k}}{y_{k}}\right)-L\right| \geq \epsilon\right\}\right| \geq \delta \text { implies that } \frac{1}{\lambda_{n}} \sum_{k \in I_{n}}\left|\left(\frac{x_{k}}{y_{k}}\right)-L\right| \geq \epsilon \delta .
$$


Thus we have the inclusion

$$
\left\{n \in \mathbb{N}: \frac{1}{\lambda_{n}}\left|\left\{k \in I_{n}:\left|\left(\frac{x_{k}}{y_{k}}\right)-L\right| \geq \epsilon\right\}\right| \geq \delta\right\} \subseteq\left\{n \in \mathbb{N}: \frac{1}{\lambda_{n}} \sum_{k \in I_{n}}\left|\left(\frac{x_{k}}{y_{k}}\right)-L\right| \geq \epsilon \delta\right\} .
$$

Since $x \sim^{[V, \lambda]^{L_{(\mathscr{A})}}} y$, it follows by definition that the set on the right side belongs to $\mathscr{I}$ which immediately implies

$$
\left\{n \in \mathbb{N}: \frac{1}{\lambda_{n}}\left|\left\{k \in I_{n}:\left|\left(\frac{x_{k}}{y_{k}}\right)-L\right| \geq \epsilon\right\}\right| \geq \delta\right\} \in \mathscr{I} .
$$

This shows that $x \sim_{\lambda}^{L}(\mathscr{I}) y$.

We next give an example to show that the inclusion $[V, \lambda]^{L}(\mathscr{I}) \subset S_{\lambda}^{L}(\mathscr{I})$ is proper. Consider the sequences $x=\left(x_{k}\right)$ and $y=\left(y_{k}\right)$ defined by

$$
x_{k}=\left\{\begin{array}{l}
k, \text { for } n-\left[\sqrt{\lambda_{n}}\right]+1 \leq k \leq n, \\
0, \text { otherwise }
\end{array}\right.
$$

and $y_{k}=1$ for all $k$. Then, clearly $x=\left(x_{k}\right) \notin \ell_{\infty}$. We have, for every $\epsilon>0(0<\epsilon \leq 1)$

$$
\frac{1}{\lambda_{n}}\left|\left\{k \in I_{n}:\left|\left(\frac{x_{k}}{y_{k}}\right)-0\right| \geq \epsilon\right\}\right|=\frac{1}{\lambda_{n}}\left|\left\{k \in I_{n}: n-\left[\sqrt{\lambda_{n}}\right]+1 \leq k \leq n\right\}\right|=\frac{\left[\sqrt{\lambda_{n}}\right]}{\lambda_{n}} \rightarrow 0
$$

as $n \rightarrow \infty$, it follows for every $\delta>0$, we can find a positive integer $m(\delta)$ such that

$$
\left\{n \in \mathbb{N}: \frac{1}{\lambda_{n}}\left|\left\{k \in I_{n}:\left|\left(\frac{x_{k}}{y_{k}}\right)-0\right| \geq \epsilon\right\}\right| \geq \delta\right\} \subset\{1,2,3, \ldots,(m-1)\} .
$$

Since $\mathscr{I}$ is admissible so the set on the right side belongs to $\mathscr{I}$, which immediately implies

$$
\left\{n \in \mathbb{N}: \frac{1}{\lambda_{n}}\left|\left\{k \in I_{n}:\left|\left(\frac{x_{k}}{y_{k}}\right)-0\right| \geq \epsilon\right\}\right| \geq \delta\right\} \in \mathscr{I} .
$$

This shows that $x \sim_{\lambda}^{0}(\mathscr{I}) y$. On the other side, the fact

$$
\frac{1}{\lambda_{n}} \sum_{k \in I_{n}}\left|\left(\frac{x_{k}}{y_{k}}\right)-0\right| \rightarrow \infty \text { as } n \rightarrow \infty
$$

implies that the sequences $x=\left(x_{k}\right)$ and $y=\left(y_{k}\right)$ fail to be a members of $[V, \lambda]^{0}(\mathscr{I})$.

(ii) If $x=\left(x_{k}\right), y=\left(y_{k}\right) \in \ell_{\infty}$ such that $x \sim_{\lambda}^{L}(\mathscr{I}) y$. Then we can find a real number $M$ (say) such that $\left|\left(\frac{x_{k}}{y_{k}}\right)-L\right| \leq M$ for all $k \in \mathbb{N}$.

Given $\epsilon>0$, we have

$$
\frac{1}{\lambda_{n}} \sum_{k \in I_{n}}\left|\left(\frac{x_{k}}{y_{k}}\right)-L\right|=\frac{1}{\lambda_{n}} \sum_{k \in I_{n} \text { and }\left|\left(\frac{x_{k}}{y_{k}}\right)-L\right| \geq \epsilon}\left|\left(\frac{x_{k}}{y_{k}}\right)-L\right|+\frac{1}{\lambda_{n}} \sum_{k \in I_{n} \text { and }\left|\left(\frac{x_{k}}{y_{k}}\right)-L\right|<\epsilon}\left|\left(\frac{x_{k}}{y_{k}}\right)-L\right|
$$




$$
\leq \frac{M}{\lambda_{n}} \mid\left\{k \in I_{n}:\left|\left(\frac{x_{k}}{y_{k}}\right)-L\right| \geq \frac{\epsilon}{2} \mid+\frac{\epsilon}{2} .\right.
$$

Thus, if we denote the sets

$$
B(\epsilon)=\left\{n \in \mathbb{N}: \frac{1}{\lambda_{n}} \sum_{k \in I_{n}}\left|\left(\frac{x_{k}}{y_{k}}\right)-L\right| \geq \epsilon\right\}
$$

and

$$
A(\epsilon)=\left\{n \in \mathbb{N}: \frac{1}{\lambda_{n}} \mid\left\{k \in I_{n}:\left|\left(\frac{x_{k}}{y_{k}}\right)-L\right| \geq \frac{\epsilon}{2} \mid \geq \frac{\epsilon}{2 M}\right\},\right.
$$

then we have the inclusion $B(\epsilon) \subset A(\epsilon)$. Since $x \sim S_{\lambda}^{L}(\mathscr{I}) y$, it follows that $A(\epsilon) \in \mathscr{I}$ and therefore $B(\epsilon) \in \mathscr{I}$. This shows that $x \sim^{[V, \lambda]^{L}(\mathscr{G})} y$.

Further, we have

$$
\begin{aligned}
\frac{1}{n} \sum_{k=1}^{n}\left|\left(\frac{x_{k}}{y_{k}}\right)-L\right| & =\frac{1}{n} \sum_{k=1}^{n-\lambda_{n}}\left|\left(\frac{x_{k}}{y_{k}}\right)-L\right|+\frac{1}{n} \sum_{n-\lambda_{n}+1}^{n}\left|\left(\frac{x_{k}}{y_{k}}\right)-L\right| \\
& \leq \frac{1}{\lambda_{n}} \sum_{k=1}^{n-\lambda_{n}}\left|\left(\frac{x_{k}}{y_{k}}\right)-L\right|+\frac{1}{\lambda_{n}} \sum_{k \in I_{n}}\left|\left(\frac{x_{k}}{y_{k}}\right)-L\right| \\
& \leq \frac{2}{\lambda_{n}} \sum_{k \in I_{n}}\left|\left(\frac{x_{k}}{y_{k}}\right)-L\right| .
\end{aligned}
$$

Thus for any $\delta>0$,

$$
\left\{n \in \mathbb{N}: \frac{1}{n} \sum_{k=1}^{n}\left|\left(\frac{x_{k}}{y_{k}}\right)-L\right| \geq \delta\right\} \subseteq\left\{n \in \mathbb{N}: \frac{1}{\lambda_{n}} \sum_{k \in I_{n}}\left|\left(\frac{x_{k}}{y_{k}}\right)-L\right| \geq \frac{\delta}{2}\right\} .
$$

Since $x \sim^{[V, \lambda]^{L_{(\mathscr{A})}}} y$, it follows by definition of an ideal that $x \sim^{[C, 1]^{L_{(\mathscr{I})}}} y$.

(iii) This immediately follows from (i) and (ii).

Theorem 3.2. Let $\mathscr{I} \subseteq \mathscr{P}(\mathbb{N})$ be an admissible ideal and $\lambda=\left(\lambda_{n}\right)$ be any non-decreasing real sequence tending to $\infty$ with $\lambda_{n+1} \leq \lambda_{n}+1$ for all $n \in \mathbb{N}, \lambda_{1}=1$, then we have the following.

(i) $S^{L}(\mathscr{I}) \subset S_{\lambda}^{L}(\mathscr{I})$ if $\liminf _{n \rightarrow \infty}\left(\frac{\lambda_{n}}{n}\right)>0$.

(ii) If $\liminf _{n \rightarrow \infty}\left(\frac{\lambda_{n}}{n}\right)=0$, then $S^{L}(\mathscr{I}) \nsubseteq S_{\lambda}^{L}(\mathscr{I})$.

Proof. Suppose $x=\left(x_{k}\right)$ and $y=\left(y_{k}\right)$ be two sequences such that $x \sim^{S^{L}(\mathscr{I})} y$. For a given $\epsilon>0$, we have

$$
\left\{k \leq n:\left|\frac{x_{k}}{y_{k}}-L\right| \geq \epsilon\right\} \supseteq\left\{k \in I_{n}:\left|\frac{x_{k}}{y_{k}}-L\right| \geq \epsilon\right\}
$$

Therefore

$$
\frac{1}{n}\left|\left\{k \leq n:\left|\frac{x_{k}}{y_{k}}-L\right| \geq \epsilon\right\}\right| \geq \frac{1}{n}\left|\left\{k \in I_{n}:\left|\frac{x_{k}}{y_{k}}-L\right| \geq \epsilon\right\}\right|=\left(\frac{\lambda_{n}}{n}\right)\left(\frac{1}{\lambda_{n}}\right)\left|\left\{k \in I_{n}:\left|\frac{x_{k}}{y_{k}}-L\right| \geq \epsilon\right\}\right| .
$$


Let $\liminf _{n \rightarrow \infty}\left(\frac{\lambda_{n}}{n}\right)=a>0$. By virtue of definition, the set $A\left(\frac{a}{2}\right)=\left\{n \in \mathbb{N}: \frac{\lambda_{n}}{n}<\frac{a}{2}\right\}$ is finite and therefore belongs to $\mathscr{I}$ as $\mathscr{I}$ is admissible. Furthermore, for any $n \in \mathbb{N}$ either $n \in A\left(\frac{a}{2}\right)$ or $n \in A^{C}\left(\frac{a}{2}\right)$.

If $n \in A^{C}\left(\frac{a}{2}\right)$, then from (1), we have

$$
\frac{1}{n}\left|\left\{k \leq n:\left|\frac{x_{k}}{y_{k}}-L\right| \geq \epsilon\right\}\right| \geq\left(\frac{a}{2}\right) \frac{1}{\lambda_{n}}\left|\left\{k \in I_{n}:\left|\frac{x_{k}}{y_{k}}-L\right| \geq \epsilon\right\}\right| .
$$

Thus for any $\delta>0$,

$$
\left\{n \in \mathbb{N}: \frac{1}{\lambda_{n}}\left|\left\{k \in I_{n}:\left|\frac{x_{k}}{y_{k}}-L\right| \geq \epsilon\right\}\right| \geq \delta\right\} \subseteq\left\{n \in \mathbb{N}: \frac{1}{n}\left|\left\{k \leq n:\left|\frac{x_{k}}{y_{k}}-L\right| \geq \epsilon\right\}\right| \geq \frac{a \delta}{2}\right\} \cup A\left(\frac{a}{2}\right)
$$

Since $x \sim^{L}(\mathscr{I}) y$ and $A\left(\frac{a}{2}\right) \in \mathscr{I}$, it follows by definition of an ideal that

$$
\left\{n \in \mathbb{N}: \frac{1}{\lambda_{n}}\left|\left\{k \in I_{n}:\left|\left(\frac{x_{k}}{y_{k}}\right)-L\right| \geq \epsilon\right\}\right| \geq \delta\right\} \in \mathscr{I} .
$$

This shows that $x \sim_{\lambda}^{L}(\mathscr{I}) y$.

(ii) Suppose that $\liminf _{n \rightarrow \infty}\left(\frac{\lambda_{n}}{n}\right)=0$. As in [3; p. 510], we can choose a subsequence $(n(j))_{j=1}^{\infty}$ such that $\frac{\lambda_{n(j)}}{n(j)}<\frac{1}{j}$. Define the sequences $x=\left(x_{k}\right)$ and $y=\left(y_{k}\right)$ as follows,

$$
x_{i}=\left\{\begin{array}{l}
1, \text { for } i \in I_{n(j)}, j=1,2,3, \ldots \\
0, \text { otherwise }
\end{array}\right.
$$

and $y_{i}=1$ for all $i \in \mathbb{N}$. Then, $x \sim^{S^{L}} y$ and hence by the admissibility of the ideal $\mathscr{I}, x \sim^{S^{L}(\mathscr{I})}$ $y$. On the other side $x \sim^{[V, \lambda]^{L(\mathscr{A})}} y$ is not satisfied and therefore Theorem 3.2 (ii) implies that $x \sim_{\lambda}^{L}(\mathscr{I}) y$ is not satisfied.

Theorem 3.3. Let $\mathscr{I} \subseteq \mathscr{P}(\mathbb{N})$ be an admissible ideal and $\lambda=\left(\lambda_{n}\right)$ be any non-decreasing real sequence tending to $\infty$ with $\lambda_{n+1} \leq \lambda_{n}+1, \lambda_{1}=1$. If $\lim _{n \rightarrow \infty}\left(\frac{\lambda_{n}}{n}\right)=1$, then $S_{\lambda}^{L}(\mathscr{I}) \subset S^{L}(\mathscr{I})$.

Proof. Suppose that $\lim _{n \rightarrow \infty}\left(\frac{\lambda_{n}}{n}\right)=1$ and there exist sequences $x=\left(x_{k}\right)$ and $y=\left(y_{k}\right)$ such that $x \sim S_{\lambda}^{L}(\mathscr{I}) y$. We prove that $x \sim^{S^{L}(\mathscr{I})} y$. Let $\delta>0$ be given. Since $\lim _{n \rightarrow \infty}\left(\frac{\lambda_{n}}{n}\right)=1$, so we can find a positive integer $m=m(\delta)$ such that

$$
\left|\frac{\lambda_{n}}{n}-1\right|<\frac{\delta}{2} \text { for all } n \geq m .
$$

Let $A\left(\frac{\delta}{2}\right)=\left\{n \in \mathbb{N}:\left|\frac{\lambda_{n}}{n}-1\right| \geq \frac{\delta}{2}\right\}$ and $A^{C}\left(\frac{\delta}{2}\right)=\left\{n \in \mathbb{N}:\left|\frac{\lambda_{n}}{n}-1\right|<\frac{\delta}{2}\right\}$. Clearly $A\left(\frac{\delta}{2}\right) \subseteq\{1,2,3, \ldots(m-$ $1)\}$ and $A\left(\frac{\delta}{2}\right) \cup A^{C}\left(\frac{\delta}{2}\right)=\mathbb{N}$. Moreover, for any $n \in A^{C}\left(\frac{\delta}{2}\right)$ and any $\epsilon>0$ we have

$$
\frac{1}{n}\left|\left\{k \leq n:\left|\frac{x_{k}}{y_{k}}-L\right| \geq \epsilon\right\}\right|=\frac{1}{n}\left|\left\{k \leq n-\lambda_{n}:\left|\frac{x_{k}}{y_{k}}-L\right| \geq \epsilon\right\}\right|+\frac{1}{n}\left|\left\{k \in I_{n}:\left|\frac{x_{k}}{y_{k}}-L\right| \geq \epsilon\right\}\right|
$$




$$
\begin{aligned}
& \leq\left(\frac{n-\lambda_{n}}{n}\right)+\frac{1}{n}\left|\left\{k \in I_{n}:\left|\frac{x_{k}}{y_{k}}-L\right| \geq \epsilon\right\}\right| \\
& \leq 1-\left(1-\frac{\delta}{2}\right)+\frac{1}{n}\left|\left\{k \in I_{n}:\left|\frac{x_{k}}{y_{k}}-L\right| \geq \epsilon\right\}\right|(\text { using (2)) } \\
& =\frac{\delta}{2}+\frac{1}{n}\left|\left\{k \in I_{n}:\left|\frac{x_{k}}{y_{k}}-L\right| \geq \epsilon\right\}\right| \leq \frac{\delta}{2}+\frac{1}{\lambda_{n}}\left|\left\{k \in I_{n}:\left|\frac{x_{k}}{y_{k}}-L\right| \geq \epsilon\right\}\right|
\end{aligned}
$$

as $\left(\lambda_{n+1} \leq \lambda_{n}+1\right.$ implies $\left.\lambda_{n} \leq n\right)$.

Thus

$$
\left\{n \in \mathbb{N}: \frac{1}{n}\left\{k \leq n:\left|\frac{x_{k}}{y_{k}}-L\right| \geq \epsilon\right\} \mid \geq \delta\right\} \subseteq\left\{n \in \mathbb{N}: \frac{1}{n}\left|\left\{k \in I_{n}:\left|\frac{x_{k}}{y_{k}}-L\right| \geq \epsilon\right\}\right| \geq \frac{\delta}{2}\right\} \cup A\left(\frac{a}{2}\right) .
$$

Since $x \sim_{\lambda}^{L}(\mathscr{I}) y$, it follows that the set on the right side belongs to $\mathscr{I}$, consequently $x \sim^{S^{L}(\mathscr{I})} y$.

\section{Acknowledgement}

We would like to express our gratitude to the referees of the paper for careful reading and useful suggestions.

\section{References}

[1] J. S. Conner, The statistical and strong p - Cesáro convergence of sequences, Analysis, 8 (1988), 47-63.

[2] H. Fast, Surla convergence statistique, Colloq. Math., 2 (1951), 241-244.

[3] A. R. Freedman, J. J. Sember, M. Raphael, Some Cesáro type summability spaces, Proc. London Math. Soc., 37(1978), 508-520.

[4] J. A. Fridy, On statistical convergence, Analysis, 5(1985), 301-313.

[5] P. Kostyrko, T. Salat and W. Wilczynski, $\mathscr{I}$-convergence, Real Anal. Exchange, 26 (2) (2000/2001), 669-686.

[6] L. Leindler, Über die de la Vallée-Pousinsche Summierbarkeit allgemeiner Orthogonal-reihen, Acta Math. Acad. Sci. Hungar., 16 (1965), 375-387.

[7] J. Li, Asymptotic equivalence of sequences and summability, Internat. J. Math. and Math. Sci., 20 (4) (1997), 749-758.

[8] I. J. Maddox, Statistical convergence in locally convex space, Math. Proc. Cambridge Philos. Soc., 104 (1988), 141-145.

[9] M. Marouf, Asymptotic equivalence and summability, Internat. J. Math. Math. Sci., 16 (4) (1993), 755-762.

[10] M. Mursaleen, $\lambda$-statistical convergence, Math. Slovaca, 50 (1) (2000), 111-115.

[11] R. F. Patterson, On asymptotically statistically equivalent sequences, Demonstratio Math., 36 (1) (2003), 149153.

[12] R. F. Patterson, E. Savaś, On asymptotically lacunary statistically equivalent sequences, Thai J. Math., 4(2)(2006), 267-272.

[13] I. P. Pobyvanets, Asymptotic equivalence of some linear transformation defined by a non-negative matrix and reduced to generalized equivalence in the sense of Cesáro and Abel, Mat. Fiz., 28 (1980), 83-87, 123.

[14] D. Rath and B. C. Tripathy, Matrix maps on sequence spaces associated with sets of integers, Indian Jour. Pure Appl. Math., 27 (2) (1996), 197-206.

[15] T. Šalát, On statistically convergent sequences of real numbers, Math. Slovaca., 30 (1980), 139-150.

[16] E. Savaś, P. Das, A generalized statistical convergence via ideals, Appl. Math. Lett., 24 (2011), 826-830. 
[17] I. J. Schoenberg, The integrability of certain functions and related summability methods, Amer. Math. Monthly., 66 (1959), 361-375.

[18] B. C. Tripathy, Matrix transformations between some class of sequences, J. Math. Anal. Appl., 206 (1997), $448-450$.

[19] B. C. Tripathy and M. Sen, On generalized statistically convergent sequences, Indian Jour. Pure Appl. Math., 32 (11) (2001), 1689-1694.

[20] B. C. Tripathy, On generalized difference paranormed statistically convergent sequences, Indian Jour. Pure Appl. Math., 35 (5) (2004), 655-663.

[21] B. C. Tripathy and M. Sen, Characterization of some matrix classes involving paranormed sequence spaces, Tamkang J. Math., 37 (2) (2006), 155-162.

[22] B. C. Tripathy and B. Sarma, Statistically convergent difference double sequence spaces, Acta Math. Sin., 24 (5) (2008), 737-742.

[23] B. C. Tripathy and A. Baruah, Lacunary statistically convergent and lacunary strongly convergent generalized difference sequences of fuzzy real numbers, Kyungpook Math. J., 50 (2010), 565-574.

[24] B. C. Tripathy and B. Hazarika, $\mathscr{I}$-convergent sequence spaces associated with multiplier sequence spaces, Math. Inequal. Appl., 11 (3) (2008), 543-548.

[25] B. C. Tripathy and B. Hazarika, Paranormed $\mathscr{I}$-convergent sequences spaces, Math. Slovaca., 59 (4) (2009), 485-494.

[26] B. C. Tripathy and S. Mahanta, On $\mathscr{I}$-acceleration convergence of sequences, J. Franklin Inst., 347 (2010), 591-598.

[27] B. C. Tripathy and B. Hazarika, $\mathscr{I}$-convergent sequences spaces defined by Orlicz function, Acta Math. Appl. Sin., 27 (1) (2011) 149-154.

[28] B. C. Tripathy and B. Hazarika, $\mathscr{I}$-monotonic and $\mathscr{I}$-convergent sequences, Kyungpook Math. J., 51(2) (2011), 233-239.

Department of Mathematics, Haryana College of Technology and Management, Kaithal-136027, Haryana, India.

E-mail: vjy_kaushik@yahoo.com 